La Revue

des Droits

de l'Homme

\section{La Revue des droits de l'homme}

Revue du Centre de recherches et d'études sur les droits fondamentaux

Actualités Droits-Libertés | 2020

\title{
Peur, attentes et dénuement. La France est condamnée pour traitement dégradant des demandeurs d'asile
}

À propos de la décision de la Cour EDH, 2 juillet 2020, N.H. \& autres C. France

\section{Marie-Laure Basilien-Gainche}

\section{(2) OpenEdition}

\section{Electronic version}

URL: http://journals.openedition.org/revdh/10408

DOI: $10.4000 /$ revdh. 10408

ISSN: 2264-119X

\section{Publisher}

Centre de recherches et d'études sur les droits fondamentaux

\section{Electronic reference}

Marie-Laure Basilien-Gainche, «Peur, attentes et dénuement. La France est condamnée pour traitement dégradant des demandeurs d'asile », La Revue des droits de l'homme [Online], Actualités Droits-Libertés, Online since 14 July 2020, connection on 06 November 2020. URL : http:// journals.openedition.org/revdh/10408; DOI : https://doi.org/10.4000/revdh.10408

This text was automatically generated on 6 November 2020 .

Tous droits réservés 


\title{
Peur, attentes et dénuement. La France est condamnée pour traitement dégradant des demandeurs d'asile
}

À propos de la décision de la Cour EDH, 2 juillet 2020, N.H. \& autres c. France

\author{
Marie-Laure Basilien-Gainche
}

1 Dans une décision rendue le 2 juillet 2020, la Cour européenne des droits de l'homme (ci-après Cour EDH) a condamné la France pour violation de l'article 3 de la Convention européenne des droits de l'homme (ci-après CEDH) dans trois des cinq affaires qui étaient jointes : elle estime que les autorités françaises ont manqué à leurs obligations prévues par le droit interne à l'encontre des requérants, ressortissants de pays tiers ayant sollicité la protection internationale, à savoir N.H. de nationalité russe né en 1987 (Req. $n^{\circ}$ 28820/13), K.T. également de nationalité russe né en 1990 (Req. $n^{\circ}$ 75547/13) et A.J. de nationalité iranienne né en 1974 (Req. no 13114/15) ${ }^{1}$. Pour la Cour, qui s'appuie sur les tierces interventions de la Coordination nationale pour le droit d'asile et du Défenseur des Droits, les autorités françaises « doivent être tenues pour responsables des conditions dans lesquelles ils se sont trouvés pendant des mois, vivant dans la rue, sans ressources, sans accès à des sanitaires, ne disposant d'aucun moyen de subvenir à leurs besoins essentiels et dans l'angoisse permanente d'être attaqués et volés »; et les requérants doivent être regardés comme «victimes d'un traitement dégradant témoignant d'un manque de respect pour leur dignité » (\$184).

Depuis la décision Khan du 28 février 2019 (Cour EDH, 28 février 2019, Khan c. France, Req. $\left.\mathrm{n}^{\circ} 12267 / 16\right)^{2}$, la France est pour la cinquième fois condamnée par la Cour EDH (cinquième section) pour violation de l'article 3 de la Convention, selon lequel « nul ne peut être soumis à la torture ni à des peines ou traitements inhumains ou dégradants ». Une affaire concernait la défaillance du système de protection de l'enfance qui n'était pas parvenu à empêcher qu'un mineur subisse de graves abus et sévices et finisse par 
en mourir (Cour EDH, 4 juin 2020, Association Innocence en danger et Association Enfance et partage c. France, Req. $\mathrm{n}^{\circ} 15343 / 15$ \& 16806/15). Ont été mis par ailleurs en lumière les traitements inhumains et dégradants subis par les personnes détenues, d'une part à raison de la surpopulation carcérale (30 janvier 2020, J.M.B. c. France, Req. $n^{\circ}$ 9671/15, 9674/15 \& 9679/15), d'autre part à raison des usages disproportionnés de la force par des surveillants pénitentiaires (5 décembre 2019, J.M. c. France, Req. $\left.n^{\circ} 71670 / 14\right)$. Quant à la situation des migrants, elle est au cœur des deux dernières condamnations de la France pour violation de l'article 3 de la Convention, qu'il s'agisse du placement de mineurs isolés de 3 et 5 ans en centre de rétention administrative (CRA) et de leur rattachement arbitraire à un adulte tiers aux fins de les éloigner du territoire de Mayotte (Cour EDH, 25 juin 2020, Moustahi c. France, Req. n 9347/14), ou qu'il s'agisse de l'extrême dénuement des demandeurs d'asile qui se trouvent sans hébergement et sans ressources (Cour EDH, 2 juillet 2020, N.H. \& alii c. France, Req. $\left.n^{\circ} 28820 / 13,75547 / 13 \& 13114 / 15\right)$.

\section{I/- Un abandon au dénuement constitutif d'un traitement dégradant}

3 Dans cette affaire qui porte sur des faits remontant à plus de sept ans, l'enjeu était de savoir si la situation des requérants était comparable ou non à celle de ce ressortissant afghan demandeur d'asile qui avait été livré à lui-même et délaissé dans le plus extrême dénuement par les autorités helléniques, ce qui avait conduit la Cour EDH a condamné d'une part la Grèce et d'autre part la Belgique qui avait opéré le transfert du requérant en application du règlement Dublin II (Cour EDH, GC, 21 janvier 2011, M.M.S. c. Belgique \& Grèce, Req. $n^{\circ}$ 30696/09). Certes, la prohibition posée par l'article 3 revêt en ellemême un caractère absolu ( $\$ 156 \& 157)$. Mais, pour déterminer si la gravité des faits atteint le seuil minimal requis pour que soient constitués des traitements dégradants, le juge doit effectuer une appréciation par nature relative qui « dépend de l'ensemble des données de la cause, et notamment, de la durée du traitement, de ses effets physiques ou mentaux, ainsi que, parfois, du sexe, de l'âge et de l'état de santé de la victime » (\$158).

Quelques indications étaient présentes dans la jurisprudence antérieure de la Cour: constituait un traitement dégradant le fait de délaisser pendant quelque six mois dans la «jungle» de Calais un mineur afghan de 11 ans (Cour EDH, 28 février 2019, Khan c. France, Req. $\left.n^{\circ} 12267 / 16\right)$. Il est à noter ici que le Conseil d'État semble avoir intégré les exigences rappelées par la Cour dans cet arrêt, puisque, dans une décision du 8 juillet 2020, il affirme que «Le manquement ainsi commis par le préfet de la Loire-Atlantique à son obligation d'assurer à Mme A... et à ses deux enfants, selon leurs besoins et leurs ressources, des conditions d'accueil comprenant l'hébergement, la nourriture et l'habillement est constitutif d'une faute de nature à engager la responsabilité de l'État " (CE, 8 juillet 2020, Req. 425310, $§ 7$ ). En revanche, la Cour a apprécié que n'était pas constitutif d'un traitement dégradant le fait de laisser 40 nuits sans hébergement un mineur isolé étranger, considéré comme majeur par les autorités françaises (Cour EDH, 10 octobre 2019, M.D. c. France, Req. $n^{\circ}$ 50376/13). Que pouvait-il en être concernant N.H., K.T., et A.J.qui étaient regardés par les autorités «comme de jeunes majeurs isolés, en bonne santé et sans charge de famille » (§ 186)? 
Ils étaient âgés au moment des faits de 26, 39 et 23 ans respectivement ; sont restés sans ressources durant 262,185 , et 133 jours; ont vécu dans la rue pendant 262, 270 et 170 jours; sont demeurés dans la peur d'être arrêtés et renvoyés vers leur pays d'origine, faute de disposer des documents pouvant justifier leur qualité de demandeur d'asile durant 95,131 et 90 jours ( $\$ 172$ - le juge renvoie aux tierces interventions de la Coordination nationale pour le droit d'asile et du Défenseur des Droits, ainsi qu'aux les rapports publiés en avril 2013 et en avril 2014 par l'Inspection générale des finances, l'Inspection générale des affaires sociales et l'Inspection générale de l'administration, d'une part, et par le Comité d'évaluation et de contrôle des politiques publiques de l'Assemblée nationale, d'autre part). La Cour estime que le degré de gravité requis pour constater un traitement dégradant est atteint dans ces trois cas. Tel n'est pas le cas dans l'affaire S.G., le requérant ayant perçu l'aide de l'État (allocation temporaire d'attente ATA) 63 jours après son premier rendez-vous en préfecture ( $\$ 186)$. Une question demeure : à partir de combien de jours sans ressources, à la rue, dans le dénuement et dans la peur, la situation devient-elle constitutive d'un traitement dégradant? Plus de 63 jours et moins de 170 est une réponse bien incertaine qui est relative de surcroît.

Bien évidemment, la Cour aborde les raisons pour lesquelles ces demandeurs d'asile se sont trouvés dans une telle situation de dénuement. Elle relève que les requérants n'étaient pas autorisés à exercer une activité professionnelle pendant la durée de la procédure, qu'ils «dépendaient entièrement de la prise en charge matérielle et financière prévue par le droit national qui devait leur être accordée tant qu'ils étaient autorisés à demeurer sur le territoire en qualité de demandeurs d'asile » (\$167), et que faute de bénéficier des aides prévues " pour répondre à leurs besoins fondamentaux, ils n'ont pu, pendant les périodes où ils vécurent à la rue sans ressources financières, que s'en remettre à la générosité de particuliers ou à l'aide d'associations caritatives fondées sur le bénévolat » ( $\$ 179)$.

\section{II/- Une procédure de demande d'asile avec un jeu de délais aux effets pervers}

$7 \quad$ Et la Cour de relever longuement le jeu des délais qui ont conduit et conduisent encore nombre de demandeurs d'asile à vivre dans la plus grande précarité :

- délai concernant l'examen de la demande d'admission au séjour au titre de l'asile, qui court à compter du moment où le demandeur se présentait à la préfecture avec une domiciliation et les documents requis pour enregistrer sa demande d'asile et l'autoriser à séjourner régulièrement, qui devait être de 15 jours selon l'article R. 742-1 du CESEDA, mais qui était en pratique de trois à cinq mois dans les faits, ce qui ajoute le délai pour obtenir ce premier rendez-vous à la préfecture ;

- délai concernant l'enregistrement de la demande d'asile par la préfecture - qui doit être déposée dans les 21 jours suivant la sollicitation de l'asile - (95 jours pour N.H. et 131 jours pour K.T.) ;

- délai concernant l'obtention du récépissé de dépôt de la demande d'asile (28 jours dans le cas de S.G.) ;

- délai concernant l'obtention de l'autorisation provisoire de séjour au titre de l'asile (90 jours dans le cas de A.J.) ; 
- délai concernant le versement de l'Allocation temporaire d'attente ATA (qui a été remplacée par l'Allocation pour demandeur d'asile avec l'adoption de la loi $\mathbf{n}^{\circ}$ 2015-925 du 29 juillet 2015 relative à la réforme du droit d'asile) dont la demande est conditionnée par la production devant Pôle emploi, d'une autorisation de séjour au titre de l'asile et d'une preuve de dépôt effectif de la demande devant l'OFPRA ;

- sans oublier le délai concernant l'examen de la demande d'asile par l'OFRA voire par la CNDA (obtention par N.H. de la protection subsidiaire 229 jours après son arrivée en France, reconnaissance du statut de réfugié pour A.J. 188 jours après sa première convocation à la préfecture de police, rejet de la demande d'asile après 448 jours pour S.G. et 472 jours pour K.T.).

8 On aimerait bien savoir si la mise en place d'un guichet unique à Paris a effectivement changé les choses pour les demandeurs d'asile : les difficultés d'accès à la plateforme téléphonique mise en place par l'office français de l'immigration et de l'intégration (OFII) pour prendre rendez-vous dans les structures de premier accueil des demandeurs d'asile (SPADA) qui sont chargés de délivrer les convocations dans les guichets uniques des demandeurs d'asile (GUDA) sont autant d'entraves à l'accès au droit d'asile que la Cimade a dénoncées.

Car l'enchainement de ces délais, dont les durées fixées par la loi s'avèrent beaucoup longues dans la réalité, révèle la complexité du parcours du demandeur d'asile qui plonge l'observateur dans une perplexité certaine et qui abandonne l'intéressé dans une précarité délétère. Et la Cour de préciser, non sans une certaine ingénuité, «qu'il ne lui appartient aucunement de se prononcer sur ces délais mais qu'il lui revient en revanche d'examiner leur incidence sur la situation des requérants afin de déterminer si le seuil de gravité prévu par l'article 3 de la Convention était atteint» ( $\$ 170)$. L'incidence du jeu des délais est bien le délaissement des demandeurs d'asile dans un dénuement dont la gravité atteint le seuil pour constituer un traitement dégradant, alors que "l'obligation de fournir un hébergement ou des conditions matérielles décentes aux demandeurs d'asile démunis fait à ce jour partie du droit positif et pèse sur les autorités de l'État défendeur concerné en vertu des termes mêmes de la législation nationale qui transpose le droit de l'Union européenne, à savoir la " directive Accueil » ( $(\S)$.

\section{III/- Le renvoi à une obligation de résultat posé par le droit interne}

10 Certes, la Cour se veut rassurante quand elle rappelle que «l'article 3 ne saurait être interprété comme obligeant les Hautes Parties contractantes à garantir un droit au logement à toute personne relevant de leur juridiction » et comme imposant "un devoir général de fournir aux réfugiés une assistance financière pour que ceux-ci puissent maintenir un certain niveau de vie » (\$160). Mais elle renvoie fermement les autorités françaises au respect de leur droit national : elles n'ont pas respecté les délais dans lesquels les demandes d'asile doivent être enregistrées ( 3 jours étendus à 10 jours en cas de nombreuses demandes simultanées au titre de l'article L. 741-1 du CESEDA), délais qui ont été considérés par le Conseil d'État comme imposant une obligation de

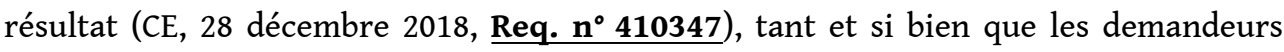
d'asile se sont trouvés dans une situation de dénuement. Autrement dit, la Cour apprécie l'existence d'une violation de l'article 3 de la Convention à l'aune des 
dispositions juridiques en vigueur et de leur portée contraignante, de telle sorte qu'elle apparaît bien plus que le juge national la garante de l'ordre juridique interne.

En effet, la Cour EDH affirme clairement que l'obligation de résultat de respecter les délais fixés par le droit interne ne peut être relativisée en raison de l'absence d'urgence humanitaire ( $\$ 182$ «les faits qui lui sont soumis s'inscrivent dans une hausse progressive et ne se sont donc pas déroulés dans un contexte d'urgence humanitaire engendré par une crise migratoire majeure, qualifiable d'exceptionnelle, à l'origine de très importantes difficultés objectives de caractère organisationnel, logistique et structurel »), et que les autorités ne pourraient en être exonérées à raison d'un afflux croissant de migrants ( $\$ 157$ «les facteurs liés à un afflux croissant de migrants ne peuvent pas exonérer les États contractants de leurs obligations au regard de cette disposition »).

Elle réfute donc la position désormais traditionnelle du juge des référés français selon laquelle un délai de convocation de trois mois pour déposer une demande d'asile est raisonnable, si bien qu'il «être reproché à l'administration une attitude d'inertie constitutive d'une atteinte grave et manifestement illégale au droit d'asile " (affaire N.H., ordonnance du 19 avril 2013; affaire S.G., ordonnance du 7 octobre 2013; affaire A.J., ordonnance du 13 novembre 2014). Se trouve donc mise en cause la jurisprudence constante du Conseil d'État depuis l'ordonnance de 10 mai 2012 (Req. n $^{\circ}$ 358828) : pour la Cour EDH, on ne saurait admettre qu'« en cas d'afflux massif de demandeurs d'asile et au regard des moyens limités dont disposent les autorités, le délai de trois mois pour enregistrer la demande est raisonnable » et que cela n'emporte « pas d'atteinte au droit d'asile» (§ 151).

Cette position franche de la Cour découle de la prise en considération du droit national tel que modifié pour transposer des normes européennes (directives «Accueil » et « Procédures ») telles qu'elles sont interprétées par la Cour de Justice de l'Union européenne (CJUE). Ceci est particulièrement souligné par la Cour qui cite largement deux arrêts majeurs de la Cour de Luxembourg, à savoir l'arrêt Cimade \& Gisti (CJUE, 27 septembre 2012, Cimade \& Gisti c. ministre de l'Intérieur, aff. C-179/11) ${ }^{3}$ et l'arrêt Saciri (CJUE, 27 février 2014, Saciri, aff. C-79/13)4. Un tel dialogue des juges européens (la CJUE faisant pour sa part siennes les positions de la Cour EDH, notamment dans l'affaire N.S. reprenant les positions de la Cour de Strasbourg énoncées dans l'affaire M.M.S. : CJUE, GC, 21 décembre 2011, $\underline{\text { N.S. }}$, aff. jointes C-411/10 \& C-493/105) souligne combien le juge français est loin de s'engager réellement dans une application effective des obligations qui incombe aux autorités nationales du fait des dispositions du droit de l'Union et de la Convention.

\section{Conclusion}

L'arrêt rendu par la Cour EDH dans l'affaire N.H. \& autres rassure certes, mais inquiète aussi. Il rassure car le juge de Strasbourg adopte une position de garantie des droits des migrants demandeurs d'asile, alors même que sa décision très critiquable dans l'affaire N.D. et N.T. c. Espagne laisse un goût très amer devant cette in-effectivisation du principe de non-refoulement (Cour EDH, GC, 13 février 2020, Req. $\mathrm{n}^{\circ}$ 8675/15 et 8697/15). L'arrêt inquiète toutefois. La Cour rappelle bien que « les demandeurs d'asile peuvent être considérés comme vulnérables du fait de leur parcours migratoire et des 
expériences traumatiques qu'ils peuvent avoir vécues en amont» (\$ 162): on peut d'ailleurs déplorer le fait que la Cour n'ait pas simplement affirmé la vulnérabilité des demandeurs d'asile en affirmant clairement qu'ils sont considérés comme vulnérables. Mais la Cour ne dit rien de l'étrange échelle de vulnérabilité qui est mise en place par les autorités et les associations, et qui suppose de cumuler les facteurs de vulnérabilité pour pouvoir prétendre au bénéfice de conditions de vie dignes, admettant en conséquence que certains êtres humains seraient plus dignes que d'autres. Elle ne remet pas non plus en cause le sous-dimensionnement du dispositif national d'asile qui cause une insuffisance structurelle des capacités d'accueil et qui génère de manière mécanique le traitement dégradant subi par les demandeurs d'asile sans hébergement et sans ressources (Karine Parrot, Carte blanche. L'État contre les étrangers, Paris, La Fabrique, 2019).

CEDH, 2 juillet 2020, N.H. \& autres c. France.

Les Lettres « Actualités Droits-Libertés » (ADL) du CREDOF (pour s'y abonner) sont accessibles sur le site de la Revue des Droits de l'Homme (RevDH) - Contact

\section{NOTES}

1. Cet arrêt a fait l'objet d'une analyse en anglais parue sur le site du Verfassungblog: MarieLaure Basilien-Gainche, « Ask the dust. Comments on France's condemnation by the ECtHR in the N.H.case », Verfassungblog, 10 juillet 2020.

2. Marie-Laure Basilien-Gainche, «Children of Men. Comments on the ECtHR's Judgment in Khan v. France ", Verfassungblog, 12 mars 2019; voir également la campagne menée par des associations pour que soient pris en charge les mineurs non accompagnés, "À Paris, un campement de mineurs étrangers pour alerter l'Aide sociale à l'enfance », Le Monde, 30 juin 2020.

3. Marie-Laure Basilien-Gainche, « Droit d'asile (Directive 2003/09/CE) : Obligation d'octroi des conditions minimales d'accueil aux demandeurs d'asile "dublinés»", lettre ADL du 2 octobre 2012, La Revue des droits de l'homme.

4. Marie-Laure Basilien-Gainche \& Serge Slama, « Droit d'asile (Directive 2003/9/CE) : Implications concrètes du droit des demandeurs d'asile aux conditions matérielles d'accueil dignes ", lettre ADL du 4 mars 2014, La Revue des droits de l'homme.

5. Marie-Laure Basilien-Gainche, «Les gens de Dublin ont des droits », lettre ADL du 29 décembre 2011, La Revue des droits de l'homme. 


\section{ABSTRACTS}

Le 2 juillet 2020, la Cour européenne des droits de l'homme a condamné la France pour violation de l'article 3 de la Convention européenne des droits de l'homme, en ce que les autorités françaises ont laissé durant des mois des ressortissants de pays, ayant sollicité la protection internationale, sans ressources et sans hébergement. Le fait que les demandeurs d'asile aient ainsi vécu pendant une longue période dans la peur, les attentes et le dénuement est aux yeux du juge de Strasbourg constitutif d'un traitement dégradant, alors même que «l'obligation de fournir un hébergement ou des conditions matérielles décentes aux demandeurs d'asile démunis fait à ce jour partie du droit positif et pèse sur les autorités de l'État défendeur concerné, en vertu des termes mêmes de la législation nationale qui transpose le droit de l'Union européenne, à savoir la « directive Accueil » » (§).

\section{AUTHOR}

\section{MARIE-LAURE BASILIEN-GAINCHE}

Professeur des Universités en Droit Public, Université Jean Moulin Lyon III, Membre honoraire de l'Institut Universitaire de France 